\title{
COVID-19, Pathophysiology and Prospects for Early Detection in Patients with Mild Symptoms of The Controversial Virus in Underdeveloped Countries.
}

\author{
Garcés Villalá M.A ${ }^{{ }^{*}}$, Nollen, J.A¹, Rico, S.D1, Cortez Quiroga G.A², Aubone de los Ríos G.0³, Calvo \\ Guirado J.L 4 \\ 1) Department of Implant and Biomaterial Research, Fundación Corazón de Jesús, San Juan Argentina \\ 2) Servicio de Cardiología. Hospital Alto Guadaquivir (Andújar, Jaén, España) \\ 3) Jefe de Virologia. Laboratorio de Análisis Clinicos del Hospital Naval Pedro Mallo. Buenos Aires. Argentina. \\ 4) Oral and Implant Surgery, Faculty of Health Sciences, Universidad Católica San Antonio de Murcia, Murcia, Spain. \\ fundacioncorazondejesus@gmail.com \\ DOI: http://doi.org/10.29080/jhsp.v4i1.375
}

Received: Mei 2020, Accepted : Juli 2020, Published : Agustus 2020

\section{Keywords}

Pathophysiology, COVID-19, early detection symptoms

\begin{abstract}
COVID-19 has spread uniformly across the planet without distinction of hemispheres, borders, or climates. However, underdeveloped countries lack sufficient means to effectively detect and cope with the pandemic. The catastrophic economic situation forces indefinite quarantines to avoid health collapse. The scarcity of massive detection tests has led health personnel, the general population and their rulers to ignorance of the true epidemiological magnitude of SARS-CoV-2. Method: A computerized bibliographic search was performed using the Google search engine of original and review articles indexed by Scopus and WOS, in the PubMed / Medline and Cochrane databases to identify all relevant manuscripts and preprints, notices from professional societies and comments from Experts published from the start of the current epidemic related to mild COVID-19 symptoms. Tools, massive methods for detecting the disease and their low availability in underdeveloped countries were analyzed. Inexpensive and easily accessible methods for testing for the disease were discussed Result: According to the indexed publications on the incidence of hypoxemia as a symptom and parameter of the evolution of the disease, oxygen saturation of less than $93 \%$ was determined as the limit to consider the patient suspected of COVID-19. In addition, it was determined after the analysis of published studies show an average prevalence of anosmia-hyposmia as a mild symptom in 69\% of patients infected with COVID-19. Conclusion: Suspected cases of COVID-19 infection could be detected by the presence of its prevalent signs and symptoms. More studies are needed to standardize and validate the postulated screening tests.
\end{abstract}

\section{Introduction}

The new pneumonia? by coronavirus denominated COVID-19 is a contagious acute systemic infectious disease that affects the respiratory system produced by the SARS-CoV-2 virus. COVID-19 affects all types of people in different ways, without gender differences and throughout the age range, in our experience the mean age of hospitalized patients was 65.8 years (range, 8 months-103 years) presenting a very disparate clinical picture (1). The "tro-ya horse" of COVID-19 is probably the large and unknown percentage of asymptomatic patients that would multiply their contagion power exponentially. People infected with COVID-19 will develop mild to moderate symptoms, a recent study of these patients reported $85.6 \%$ and $88.0 \%$ of olfactory and gustatory dysfunctions, respectively(2).

Symptomatic patients with coronavirus may present various associated symptoms such as fever with a temperature above $37.5^{\circ} \mathrm{C}$, dry cough, fatigue, myalgia, sore throat (odino-phagia), anosmia (loss of smell), dysgeusia (loss of taste). dyspnea (shortness of breath). In addition, some patients may experience: nasal congestion, runny nose (na-salt mucus), hemoptysis (expectoration of sputum with some fresh blood from the respiratory system), diarrhea, nausea, vomiting, and conjunctivitis (3). But one study showed that 
$39.6 \%$ of 140 confirmed COVID-19 patients had gastrointestinal symptoms (4), and 10.1\% of patients initially had gastrointestinal complaints (5). On average, it takes 5 to 6 days from when someone is infected with the virus until the first symptoms appear, however, it can take up to 14 days for them to appear. People with mild symptoms who are otherwise healthy should isolate themselves, and those with fever, cough, and shortness of breath should seek immediate medical attention (3).

This disease was first discovered in December 2019 in the seafood market in South China, Hubei province, although it may have been spreading silently for years until it adopted its current lethality and adaptability. Currently, it was discovered that the SARS-CoV-2 virus genome is made up of a single strand of RNA, and is classified as a positive-stranded single-stranded RNA virus, which belongs to the genus Betacoronavirus in the Coronaviridae family $(6,7)$. This new Betacoronavirus is similar to the severe acute respiratory syndrome coronavirus (SARS-CoV) and the Middle East respiratory syndrome coronavirus (MERS-CoV); based on its genetic proximity, it probably originated from bat-derived coronaviruses with spread through an unknown intermediate mammalian host (likely wild dogs) to humans $(8,9)$. The SARS$\mathrm{CoV}-2$ viral genome was rapidly sequenced to allow diagnostic tests, epidemiological follow-up, and the development of preventive and therapeutic strategies. There are currently more than 15 million infected worldwide, which are not restricted by race and borders $(3,10,11)$.

The cellular pathogenicity mechanism of the new coronavirus remains mysterious. COVID-19 would not be pneumonia in the strict sense, because the virus does not directly kill pneumocytes, but instead triggers an "inflammatory tsunami" (an immune reaction that attacks the cells of the respiratory system similar to macrophage activation syndrome) on the vascular endothelium, producing diffuse thrombosis. This runaway response can cause more damage to the body's own cells than to the virus it is trying to defeat, and it is believed to be the main reason why the conditions of young, healthy individuals can deteriorate rapidly (12).

Histopathological study of the lungs of 38 deceased by COVID-19 (13), at the macroscopic level, showed highly congestive and edematous organs, patchy. At the histological level, it is characterized by a diffuse alveolar disease, which corresponds to the clinical images (radiography and computed axial tomography) observed in the initial and intermediate stages of the disease, with a bilateral multilobar pulmonary distribution. A fibrous phase is not observed, or this is rare, due to the short evolution time of the disease. $87 \%$ of the samples presented small thrombi $(<1 \mathrm{~mm}$ in diameter), with a distribution greater than $25 \%$ of the organ in $50 \%$ of the autopsies, and in association with high levels of D-dimer in plasma, at the level of the alveolar light, these findings are accompanied by a large acute inflammatory component (14).

These findings explain the severe hypoxemia that these patients develop, being the most serious complication of the disease, and support the hypothesis of associated coagulopathy and thrombosis, which translates or can be suspected in the face of high levels of D-dimer (15). Currently 3 possible stages of infection are recognized: 1st. Stage: Viral stage, mild or early infection. 2nd. Stage: Moderate stage with lung involvement: with hypoxia or without hypoxia. 3rd. Stage: Severe stage with systemic inflammatory response syndrome (16).

In clinical practice it is very important to determine what stage each patient is at, since the sensitivity of the diagnostic methods (PCR and serology) depends on this, the same occurs with medical treatment, and antivirals may be more useful at the beginning, antimalarials, and in intermediate-final stages the use of corticosteroids and anti-inflammatories (17).

According to the WHO, in $80 \%$ of patients the virus only causes a cold, but $5 \%$ of those infected present severe symptoms, including hyper inflammation processes, with intravascular coagulation, respiratory distress and cardiovascular events. The lung is the organ most affected by inflammation (respiratory distress), requiring in the most severe cases invasive mechanical ventilation, although the patient may also die from pulmonary thromboem-bolia, cardiogenic shock, septic shock, and acute cardiac and cerebrovascular accidents. -lar The main challenge to control this pandemic is to prevent the spread of the disease, where, beyond confinement, inform and educate the population, and develop the capacity and means to detect asymptomatic or symptomatic patients mild (18).

Detection methods of the SARS-CoV-2 virus in a hospital setting. Specific analyzes of the viral genome are performed using the Polymerase Chain Reaction (PCR) and are scarce in underdeveloped countries. Using PCR, a fragment of genetic material is located and amplified, which in the case of the coronavirus is an RNA molecule. The PCR is a test that presents a degree of complexity, so it needs trained and prepared personnel to carry it out. It has high specificity (99.9\%), since it can differentiate between two evolutionarily very close microorganisms and detect up to 10 copies of viral genetic material RNA, (Gene finder COVID-19® is the reagent used in Argentina) and is highly sensitive in the phase early infection. One-step reverse transcription real-time PCR is used to confirm the presence of COVID-19 by amplifying the virus's own RdRp, E and $\mathrm{N}$ genes according to the protocol recommended by the WHO. The $\mathrm{N}$ (from the nucleocapside) is of early appearance and the last to disappear.

Copyright (C) 2020 Garcés Villalá M.A, Nollen, J.A, Rico, S.D, Cortez Quiroga G Aubone de los Ríos GO, Calvo Guirado J.L

This work is licensed under a Creative Commons Attribution-NonCommercial-ShareAlike 4.0 International License. 
A laminar flow is required for sample processing to avoid contamination to the sample and infection of the sample to the operator. Gloves with talc are not used because talc inhibits polymerase, nitrile powderfree gloves are used.

Two dacron swabs or polyethylene microbrushes are required per patient, one nasal and one oropharyngeal. Both swabs are sent to the laboratory in a sterile tube with a stopper and immersed in $2 \mathrm{ml}$ of sterile physiological solution.

A cold chain must be maintained because the genome of this virus, being RNA, is very labile. In addition, the samples must be homogenized trying to extract all the biological material obtained by the swabs, care in their preparation is crucial to avoid reporting false negative results or obtaining invalid tests.

If the test detects RNA from the virus, the result is positive and SARS-CoV-2 infection is confirmed. Unlike PCR, rapid tests or kits for antibodies do not identify the RNA of the virus, they detect either antibodies produced against the virus in a blood sample, or virus proteins (antigens) present in respiratory samples from nasopharyngeal exudate (19).

They are only for statistical purposes and to know if the patient had contact with this virus (20). There is no certain knowledge to guarantee if the presence of antibodies indicates immunity, because it is not yet known if the patient can be reinfected.

Some reagents detect IgG (late-onset) antibodies, others Ig M (early-onset) or a combination of both. Until now the provenance of the kits speaks volumes about their sensitivity and efficiency, those of Chinese origin have very low sensitivities (30\%), however the Korean kits are more reliable.

In addition to speed, they present another very important advantage at the present time, since they can be carried out by a health professional at the home of a suspected case. These rapid diagnostic tests are based on a paper immunochromatography, that is, a platform that has the virus proteins 'stuck' to detect specific antibodies. Its operation is similar to that of pregnancy tests. This work is presented to postulate that a low cost and easily accessible screening test could be used in low-income countries that do not have massive tests to diagnose COVID-19.

\section{Methods}

A computerized bibliographic search was performed using the Google search engine of original and review articles indexed by Scopus and WOS, in the PubMed / Medline and Cochrane databases to identify all relevant manuscripts and preprints, notices from professional societies and comments from Experts published from the start of the current epidemic related to mild COVID-19 symptoms until August 23, 2020. The preprint repositories included two medRxiv articles. The combined search terms included: "COVID-19", "SARS-CoV-2", "anosmia", "hyposmia", "hypoxia". Further searches were performed based on the studies that were identified (and their references). Studies were excluded if full texts could not be obtained. Our search identified 18 peer-reviewed articles published or accepted for publication (21$32,32-37$ ) and 2 manuscript preprints (not peer-reviewed) (38,39). Tools, massive methods for detecting the disease and their low availability in underdeveloped countries were analyzed. Inexpensive and easily accessible methods for testing for the disease were discussed.

\section{Result}

Early diagnosis of COVID-19. The number of asymptomatic patients with COVID-19 is perhaps erroneously estimated between 25 to $50 \%$, this possibility alone undoubtedly justifies the use of chinstraps and frequent handwashing, although social distancing is still the best protection (40). The normal arterial blood oxygen saturation is $92 \%$ to $100 \%$. Hypoxemia is an abnormal decrease in the partial pressure of oxygen in the arterial blood below $60 \mathrm{mmHg}$. It can also be defined as an oxygen saturation less than $90.7 \%$. Patients with COVID-19 viral pneumonia may initially (mild stage or early infection) have a $10 \%$ decrease in oxygen saturation (85\% to $90 \%$ ). This "hidden initial symptom" could be compensated and unfortunately silenced. with an increase in their respiratory rate. However, patients with COVID-19 have been found to exhibit life-incompatible oxygen levels without dyspnea. Called silent hypoxemia, this pathology is especially puzzling for clinicians and is considered to defy basic biology. The coronavirus may have an idiosyncratic action on the receptors involved in chemo-sensitivity to oxygen, but well-established pathophysiological mechanisms may explain most, if not all, cases of silent hypoxemia. These mechanisms include how dyspnea and respiratory centres respond to low levels of oxygen, how prevalent tensions of carbon dioxide (PaC02) reduce the brain's response to hypoxia, the effects of disease, and age on the control of respiration, inaccuracy of pulse oximetry at low oxygen saturations and temperature-induced changes in the oxygen dissociation curve (21). 
Table 1. Publications related to mild symptoms of COVID-19.

\begin{tabular}{|c|c|c|}
\hline Author & $\begin{array}{c}\text { Publication } \\
\text { date }\end{array}$ & Mild symptom results \\
\hline Tobin MJ (21) & Aug 2020 & Silent Hypoxemia (Sp02) 62\%-76\% \\
\hline Yan CH (22) & Apr 2020 & Hyposmia-anosmia in $68 \%$ of patients \\
\hline Duca $A(23)$ & Apr 2020 & Hypoxemia $(\mathrm{SpO} 2) \leq 90 \%$ \\
\hline Dhont, S (24) & Jul 2020 & Hypoxemia (SpO2) 90\%-92\% \\
\hline Machhi, J (25) & Jul 2020 & $\begin{array}{l}\text { Hypoxemia (SpO2) } \geq 90 \% \text { + Hyposmia-anosmia in } 36,4 \\
\% \text { of patients }\end{array}$ \\
\hline $\begin{array}{l}\text { American Academy of Otolaryng Head } \\
\text { and Neck Surg (26) }\end{array}$ & March 2020 & Hyposmia-anosmia in $73 \%$ of patients \\
\hline Kaye R (27) & April 2020 & Hyposmia-anosmia in $73 \%$ of patients \\
\hline Brodwin E (28) & April 2020 & Hyposmia-anosmia in $67 \%$ of patients \\
\hline Sedaghat AR (29) & Jul 2020 & Hyposmia-anosmia in $80 \%$ of patients \\
\hline Gilani S (30) & Aug 2020 & Hyposmia-anosmia in $62,5 \%$ of patients \\
\hline Spinato G (31) & Apr 2020 & Hyposmia-anosmia in $64,4 \%$ of patients \\
\hline Lechien JR (41) & Aug 2020 & Hyposmia-anosmia in $79,6 \%$ of patients \\
\hline Yan CH (33) & Jul 2020 & Hyposmia-anosmia in $66,7 \%$ of patients \\
\hline Vaira LA (34) & Jul 2020 & Hyposmia-anosmia in $66,7 \%$ of patients \\
\hline Van Gerven L (32) & Jun 2020 & Hyposmia-anosmia in $100 \%$ of patients \\
\hline Ortiz-Prado E (35) & Aug 2020 & Hypoxemia (SpO2) $\leq 93 \%$ \\
\hline Stawicki SP (36) & May 2020 & Hypoxemia (SpO2) <90\% \\
\hline Xie J (37) & May 2020 & Hypoxemia (SpO2) <90\% \\
\hline Bagheri SHR (38) & Mar 2020 & Hyposmia-anosmia in $76.2 \%$ of patients \\
\hline Haehner A (39) & Apr 2020 & Hyposmia-anosmia in $64,7 \%$ of patients \\
\hline
\end{tabular}

Using a pulse oximeter (fig. 1), a simple medical device that can be used by the same patient, this decrease in oxygen saturation can be identified and alerted to the suspected infection. The precision of the pulse oximeters is sufficient to measure saturations greater than $90 \%$, however its accuracy decreases for saturations of lower percentage (42).

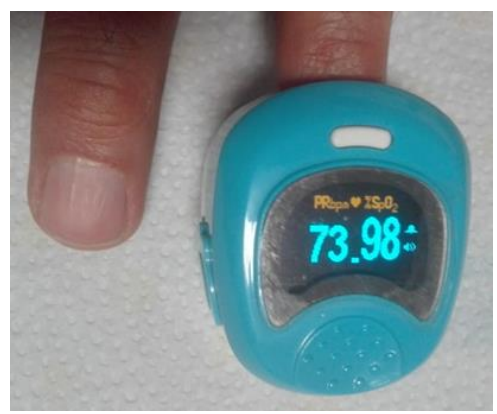

Figure 1. Pulse oximeter indicating 73 beats per minute and $98 \%$ oxygen saturation. Source: Miguel Angel Garcés Villalá Copyright 2020.

Two out of every three patients with COVID-19 present anosmia as the first - and only manifestation of the disease, even without fever, cough or sore throat, according to studies carried out on more than one hundred infected people in Wuhan. Lack of smell and taste could be a clue to alert about the presence of COVID-19 (22).

Olfactory dysfunction related to this disease can be severe and prolonged of the patients who reported COVID-19-associated loss of smell, 74\% reported resolution of anosmia with clinical resolution of the disease. Infiltration of the mucosa by CD68 + macrophages expressing the viral antigen SARS-CoV-2 may contribute to COVID-19-related olfactory dysfunction $(2,22)$.

According to the indexed publications on the incidence of hypoxemia as a symptom and parameter of the evolution of the disease, an oxygen saturation of less than $93 \%$ was determined as the limit to consider the patient suspected of COVID-19. In addition, it was determined after the analysis of published studies show an average prevalence of anosmia-hyposmia as a mild symptom in $69 \%$ of patients infected with COVID-19 (22,23). 
There are two general types of methods for measuring loss of smell: objective and subjective. Objective measures of smell (most accurate in detecting COVID-19) encompass psychophysical tests designed to measure and quantify human responses to physical stimuli. Although sparingly used in COVID19 research to date, current psycho-physical techniques include odor threshold tests to determine the lowest concentration of an odour that can be detected, odour discrimination tests to measure the ability to differentiate between odours and odour identification tests, evaluating the ability to correctly name odour qualities (43). An inexpensive early detection protocol used at a United States military base in South Korea, where people entering the army garrison smell apple cider vinegar, could be used in Latin America. In Argentina, a test (olfactory test) was developed with the "scratch and smell" method, with 10 microencapsulated fragrances (lemon, strawberry, jasmine, soap, chocolate, mandarin, vanilla, oregano, naphtha and eucalyptus) whose objective is use odours better known to the population and reduce examination costs (44).

Screening, within the framework of health systems, refers to carrying out diagnostic tests on people, in principle healthy, to distinguish those who are probably ill from those who are probably not. It is a secondary prevention activity, the objective of which is the early detection of a certain disease in order to improve its prognosis and avoid premature mortality and / or the disability associated with it. In addition, it is possible to detect mild symptoms or situations prior to the appearance of the studied disease and its early treatment will reduce its incidence and morbidity.

Screening triage by screening the suspected patient in medical and dental offices. The detection of oligosymptomatic patients is a challenge and a priority, to avoid the spread of the virus in crowded areas and at risk of confinement, such as the waiting rooms of the health system. In the health questionnaires (anamnesis), previous symptoms such as fever, cough, sore throat and if he had contact with patients are evaluated. Besides of in situ temperature control (body temperature must be less than $37^{\circ} \mathrm{C}$ ) the study of a symptom (anosmia) and an altered physiological parameter (hypoxemia) can be used synergistically as a protocol and could contribute to detect suspicious cases of infection. Measurement of hypoxemia using the pulse oximeter and detection of anosmia using olfactory tests can be used as an initial screening test for the diagnosis of a suspected COVID-19 patient. These simultaneously measured parameters are enhanced and can detect the onset of mild symptoms. Only with one of the parameters altered is it considered a suspicious case. Tests are done before starting treatment. The patient who exceeds the protocol can be treated under strict biosafety regulations. No practice is performed in the suspected patient and the immediate performance of a diagnostic test for COVID-19 is suggested.

Exams are done in the waiting room. The smell test can be carried out with coffee and vinegar (figure 2). To overcome triage, the patient must detect at least one of the aromas to rule out anosmia. Furthermore, oxygen saturation must be greater than $93 \%$ to rule out hypoxemia.

A number of biosensors, such as pulse oximetry, can be used to track the patient's status remotely. In this way, it could detect patients in the early stages of disease progression and reduce the need for patients with mild cases to remain in medical centres for prolonged periods.

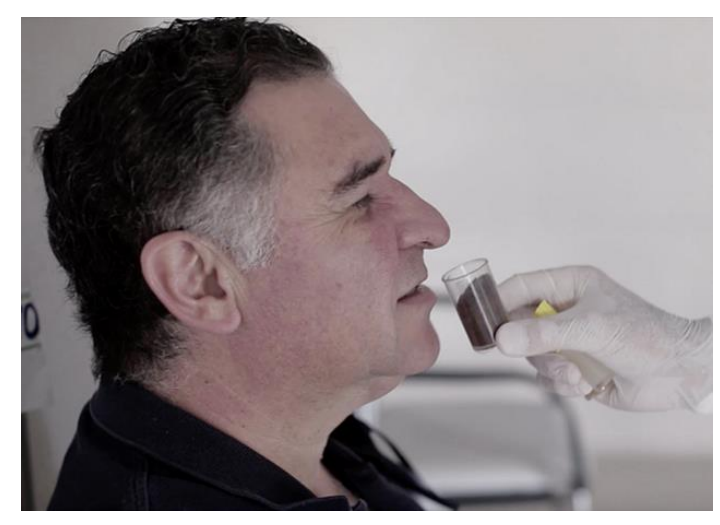

Figure 2. Patient with closed eyes detecting coffee aroma to rule out anosmia. Source: Miguel Angel Garcés Villalá Copyright 2020.

\section{Discussion}

We are facing a new disease, caused by a virus with a high contagion capacity, which manifests clinical pictures so diverse that they range from the absence of symptoms to causing a flowery inflammatory condition with high mortality. The reason why early diagnosis is one of the main challenges. 
The health and economic crisis in our environment leads us to study and understand this disease in order to develop simple, profitable and creative protocols, to improve the probability of suspicion and increase the effectiveness of diagnostic tests.

We do not currently have effective treatment, and we are awaiting the development of a reliable vaccine. But we know that if treatment of complications is delayed, the inflammation prepares the ground for the development of respiratory distress and the formation of thrombi.

COVID-19 causes not only lung injury but also serious cardiac complications, particularly in those patients with pre-existing medical conditions. Although the precise mechanism for the development of COVID-19-associated myocardial injury is not fully understood, a combination of direct and indirect pathogenic factors, such as ACE2-mediated SARS-CoV-2 infection of cardiomyocytes, hypoxia, and storm of cytokines, can contribute to the development of myocardial lesions and other adverse events that aggravate conditions and increase mortality. Close and careful monitoring of cardiac function should be emphasized to identify COVID-19-related myocardial injury early (45).

The initial stage of infection is key because it is the time when the therapy could be effective. Given the rapid pace of scientific discoveries and clinical data generated by the large number of people quickly infected with SARS-CoV-2, doctors need accurate evidence on effective medical treatments for this infection.

Therefore, leaving patients at home for several days with fever could aggravate the condition to a serious stage, increasing the risk of mortality. However, in underdeveloped countries, there are no available reagents with adequate sensitivity and specificity to ensure the accurate diagnosis of COVID-19 and the few rapid tests available could give false negatives and the infected patient could continue the chain of infections. Longitudinal serological studies are urgently needed to determine the extent and duration of immunity to SARS-CoV-2. Even in the case of apparent elimination, surveillance for SARS-CoV-2 should be maintained, as a resurgence of contagion could be possible until 2024 (46).

The proposed screening method for early detection of disease is neither a guarantee of cure nor of sure success. Like all health activities it has benefits, but also adverse effects. Although there are no potential side effects associated with the postulated tests, patients in whom early detection does not imply an improvement in their prognosis will suffer a greater period of morbidity due to the diagnostic advance that the process itself implies. Detection of abnormalities with an uncertain prognosis or precursor lesions can lead to over-diagnosis and over-treatment. For these and many other reasons that could be considered, screening is clearly not a panacea nor is it justified in itself. A screening test must be valid, reliable, simple, safe and acceptable to the target population. There must be a clear consensus regarding the process of diagnostic confirmation and evidence that treatment in the presymptomatic phase is more effective (47).

Some patients present severe hypoxemia in the absence of dyspnea, to reduce the risk of complications in such patients, a proposed solution to justify hospital admission is to control their arterial oxygenation by pulse oximetry at home. While the ease of use and low cost of pulse oximetry makes it an attractive option for identifying problems at an early stage. Counselling patients on the proper means to measure their oxygen saturation and when to seek assistance is essential to help ensure the successful implementation of the necessary monitoring programs.

The tests postulated in this work should be standardized and evaluated in systematic studies. The disadvantages found are the margin of error of the oximeter apparatus and the need to calibrate the smell test. In addition, comparative studies will be required to contrast results with rapid tests and PCR to evaluate their margin of error. However, underdeveloped countries without the operational capacity to carry out massive tests would benefit from its implementation. The protocols must be dynamic with close telephone contact in symptomatic patients to detect early clinical worsening of the patient for prompt referral to hospital emergencies. Learning on the fly, seven months after the arrival of COVID-19, do you know enough or very little? Humanity until now has always been protected by nature, perhaps this pandemic paradoxically ends up saving our planet.

\section{Conclusions}

Suspected cases of COVID-19 infection could be detected by the presence of its prevalent signs and symptoms. While large methodological differences were seen in the reviewed studies, a significant prevalence of anosmia/hyposmia is reported in patients with COVID-19, and controlled studies indicate that anosmia is more common in patients with COVID-19. Additionally, oxygen saturation less than $93 \%$ can be used as an indicator or predictor of the course of the disease.

The present work postulates that a low-cost screening test could be used in low-income countries that do not have enough tests to diagnose COVID-19. The study of a symptom (anosmia) and a physiological parameter (oxygen saturation) could contribute to detecting suspected cases of SARS-COV-2 infection. The measurement of hypoxemia using the pulse oximeter and the detection of anosmia using olfactory tests could be used as the initial diagnostic method for COVID-19. The advantages of simultaneously using the olfactory and oximetry tests are their low cost and easy access. In addition these 
parameters synergistically they are potentiated to detect the disease in the initial stages. The disadvantages found are the margin of error of the pulse oximeter and the need for calibration of the anosmia detection method. More studies are needed to standardize and validate the postulated screening tests.

\section{Acknowledgement}

We thank the Catholic University of Murcia in Spain for its unconditional support.

\section{References}

1. Registro 03/03/20-20/04/20 Hospital Alto Guadalquivir, Andújar, España.

2. Chung TW, Sridhar S, Zhang AJ. Olfactory Dysfunction in Coronavirus Disease 2019 Patients: Observational Cohort Study and Systematic Review. Open Forum Infect Dis. 2020;7(6).

3. Coronavirus disease 2019 - World Health Organization.

4. Zhang JJ, Dong X, Cao YY, Yuan YD, Yang YB, YQ Y. Clinical characteristics of 140 patients infected by SARS-CoV-2 in Wuhan, China. Allergy. 2020;

5. Wang D, Hu B, Hu C, Zhu F, Liu X, J Z. Clinical characteristics of 138 hospitalized patients with 2019 novel coronavirus-infected pneumonia in Wuhan, China. JAMA. 2020;

6. Paraskevis D, Kostaki EG, Magiorkinis G, Panayiotakopoulos G, Sourvinos G, Tsiodras S. Full-genome evolutionary analysis of the novel corona virus $(2019-\mathrm{nCoV})$ rejects the hypothesis of emergence as a result of a recent recombination event. Infect Genet Evol. 2020;79(104212).

7. Chen L, Liu W, Zhang $\mathrm{Q}, \mathrm{Xu} \mathrm{K}, \mathrm{Ye} \mathrm{G}, \mathrm{Wu} \mathrm{W}$. RNA based mNGS approach identifies a novel human coronavirus from two individual pneumonia cases in 2019 Wuhan outbreak. Emerg Microb Infect. 2020;9:313-319.

8. Fan W, Su Z, Bin Y, Yan-Mei C, Wen W, Zhi-Gang S, et al. Holmes Edward C., Zhang Yong-Zhen. A new coronavirus associated with human respiratory disease in China. Nature. 2020;579(7798):265-9.

9. Zhu N, Zhang D, Wang W. China Novel Coronavirus Investigating and Research Team. A novel coronavirus from patients with pneumonia in China, 2019. N Engl J Med. 2020;382(8).

10. Huang C, Wang Y, Li X. Clinical features of patients infected with 2019 novel coronavirus in Wuhan, China. Lancet. 2020;395:497-506.

11. Chan JF, Yuan S, Kok KH. A familial cluster of pneumonia associated with the 2019 novel coronavirus indicating person-to- person transmission: a study of a family cluster. Lancet. 2020;395:514-23.

12. Pons S, Fodil S, Azoulay E, Zafrani L. The vascular endothelium: the cornerstone of organ dysfunction in severe SARS-CoV-2 infection. Crit Care. 2020;24(1).

13. Carsana L, Sonzogni A, Nasr A. Pulmonary post-mortem findings in a series of COVID-19 cases from northern Italy: a two-centre descriptive study. The Lancet Infectious Diseases. I(ttps://doi.org/10.1016/S1473-3099(20)30434-5).

14. Mucha SR, Dugar S, McCrae K, Joseph DE, Bartholomew J, Sacha G, et al. Coagulopathy in COVID-19. Cleveland Clinic Journal of Medicine Jun. 2020;

15. Lippi G, Favaloro EJ. D-dimer is Associated with Severity of Coronavirus Disease 2019: A Pooled Analysis. Thromb Haemost. 2020 Apr 3;

16. Siddiqi HK, Mehra MR. COVID-19 illness in native and immunosuppressed states: A clinicaltherapeutic staging proposal. J Heart Lung Transplant. 2020;39(5):405-407.

17. Zhai P, Ding Y, Wu X, Long J, Zhong Y, Li Y. Epidemiología, diagnóstico y tratamiento de COVID-19. Int J Antimicrob Agents. 2020;55(5):105955.

18. Boukhris M, Hillani A, Moroni F. Cardiovascular Implications of the COVID-19 Pandemic: A Global Perspective. Can J Cardiol. 2020;36(7):1068-1080.

19. Udugama B, Kadhiresan P, Kozlowski HN. Diagnosing COVID-19: The Disease and Tools for Detection. ACS Nano. 2020;14(4):3822-3835.

20. D.P O, E.J T. Prevalence of Asymptomatic SARS-CoV-2 Infection. A Narrative Review Annals of internal medicine [Internet]. 2020 Jun 3; Available from: https://doi.org/10.7326/M20-3012

21. Tobin MJ, Laghi F, Jubran A. Why COVID-19 Silent Hypoxemia Is Baffling to Physicians. Am J Respir Crit Care Med. 2020;202(3):356-360.

22. Yan CH, Faraji F, Prajapati DP, Boone CE, DeConde AS. Association of chemosensory dysfunction and Covid-19 in patients presenting with influenza-like symptoms. International forum of allergy \& rhinology. 2020 n/a(n/a;

23. Duca A, Piva S, Focà E, Latronico N, Rizzi M. Calculated Decisions: Brescia-COVID Respiratory Severity Scale (BCRSS)/Algorithm. Emerg Med Pract. 2020;22(5 Suppl).

24. Dhont S, Derom E, Van Braeckel E. The pathophysiology of 'happy' hypoxemia in COVID-19. Respir Res. 2020;21:198. 
25. Machhi J, Herskovitz J, Senan AM. The Natural History, Pathobiology, and Clinical Manifestations of SARS-CoV-2 Infections. J Neuroimmune Pharmacol [Internet]. 2020; Available from: https://doi.org/10.1007/s11481-020-09944-5

26. American Academy of Otolaryngology Head and Neck Surgery. Coronavirus Disease 2019: Resources [Internet]. 2020. Available from: https://wwwentnetorg/content/coronavirus-disease-2019resources.

27. Kaye R, Chang CWD, Kazahaya K, Brereton J. Denneny JC 3rd. COVID-19 Anosmia Reporting Tool: Initial Findings. Otolaryngol Head Neck Surg. 2020;163(1):132-134.

28. Brodwin E. Doctors warn an inability to smell could be a symptom of COVID-19-but caution the evidence is preliminary [Internet]. 2020. Available from: https://www.statnews.com/2020/03/23/coronavirus-sense-of-smellanosmia/

29. Sedaghat AR, Gengler I, Speth MM. Olfactory Dysfunction: A Highly Prevalent Symptom of COVID-19 With Public Health Significance. Otolaryngol Head Neck Surg. Vol. 163. 2020. 12-15 p.

30. Gilani S, Roditi R, Naraghi M. COVID-19 and anosmia in Tehran, Iran. Med Hypotheses. 2020;141(109757).

31. Spinato G, Fabbris C, Polesel J. Alterations in Smell or Taste in Mildly Symptomatic Outpatients With SARS-CoV-2 Infection [published online ahead of print, 2020 Apr 22. JAMA. 2020;323(20):20892090.

32. L VG, PW H, T C, W F, C H, V H, et al. Personal protection and delivery of rhinologic and endoscopic skull base procedures during the COVID-19 outbreak. Rhinology. 2020 Jun 1;58(3):289-294.

33. Yan CH, Faraji F. DeConde AS Reply to: Self-reported olfactory loss in COVID-19: is it really a favorable prognostic factor? Int Forum Allergy Rhinol. 2020 Jul;10(7):927-928.

34. Vaira LA, Salzano G, Petrocelli M, Deiana G, Salzano FA, De Riu G. Validation of a self-administered olfactory and gustatory test for the remotely evaluation of COVID-19 patients in home quarantine. Head Neck. 2020 Jul;42(7):1570-1576.

35. Ortiz-Prado E, Simbaña-Rivera K, Gómez-Barreno L. Clinical, molecular, and epidemiological characterization of the SARS-CoV-2 virus and the Coronavirus Disease 2019 (COVID-19), a comprehensive literature review. Diagn Microbiol Infect Dis. 2020;98 1115094.

36. Stawicki SP, Jeanmonod R, Miller AC. The 2019-2020 Novel Coronavirus (Severe Acute Respiratory Syndrome Coronavirus 2) Pandemic: A Joint American College of Academic International MedicineWorld Academic Council of Emergency Medicine Multidisciplinary COVID-19 Working Group Consensus Paper. J Glob Infect Dis. 2020;12(2):47-93.

37. Xie J, Covassin N, Fan Z. Association Between Hypoxemia and Mortality in Patients With COVID-19. Mayo Clin Proc. 2020;95(6):1138-1147.

38. Coincidence of COVID-19 epidemic and olfactory dysfunction outbreak | medRxiv [Internet]. [cited 2020 Aug 26]. Available from: https://www.medrxiv.org/content/10.1101/2020.03.23.20041889v1

39. Haehner A, Draf J, Draeger S, With K, Hummel T. Predictive value of sudden olfactory loss in the diagnosis of COVID-19. 2020.

40. Byambasuren O, Cardona M, Bell K, Clark J, McLaws M-L, Glasziou P. Estimating the extent of asymptomatic COVID-19 and its potential for community transmission: systematic review and metaanalysis [Internet]. Available from: https://doi.org/10.1101/2020.05.10.20097543

41. Lechien JR, Chiesa-Estomba CM, Siati DRD, Horoi M, Bon SDL, Rodriguez A, et al. Olfactory and gustatory dysfunctions as a clinical presentation of mild-to-moderate forms of the coronavirus disease (COVID-19): a multicenter European study. Eur Arch Otorhinolaryngol. 2020;Apr 6:1-11.

42. Luks AM, Swenson ER. Pulse Oximetry for Monitoring Patients with COVID-19 at Home: Potential Pitfalls and Practical Guidance. AnnalsATS [Internet]. Available from: https://doi.org/10.1513/AnnalsATS.202005-418FR

43. Hannum ME, Ramirez VA, Lipson SJ. Objective sensory testing methods reveal a higher prevalence of olfactory loss in COVID-19 positive patients compared to subjective methods: A systematic review and meta-analysis. Preprint.

44. L H. Test para evaluación de alteraciones olfatorias: Parte I Poster. In: Encuentro Primavera 2011 Instituto Nacional de Tecnología Industrial. Argentina;

45. Geng YJ, Wei ZY, Qian HY, Huang J, Lodato R, Castriotta RJ. Pathophysiological characteristics and therapeutic approaches for pulmonary injury and cardiovascular complications of coronavirus disease 2019. Cardiovasc Pathol. 2020;47(107228).

46. Kissler SM, Tedijanto C, Goldstein E, Grad YH, Lipsitch M. Projecting the transmission dynamics of SARS-CoV-2 through the postpandemic period. Science. 2020;368(6493):860-868.

47. J.J VR. La efectividad de la detección precoz de enfermedades An Sist Sanit Navar. Vol. 30. 2007. 11$27 \mathrm{p}$. 